\title{
Nonlinear discrete fractional mixed type sum-difference equation boundary value problems in Banach spaces
}

Weidong $L V^{*}$ and Jishe Feng

\section{"Correspondence:}

Ivweidong2004@163.com

School of Mathematics and

Statistics, Longdong University,

Qingyang, Gansu 745000, P.R. China

\begin{abstract}
This paper is concerned with the existence of a unique solution to a nonlinear discrete fractional mixed type sum-difference equation boundary value problem in a Banach space. Under certain suitable nonlinear growth conditions imposed on the nonlinear term, the existence and uniqueness result is established by using the Banach contraction mapping principle. Additionally, two representative examples are presented to illustrate the effectiveness of the main result.
\end{abstract}

MSC: 26A33; 39A05; 39A10; 39A12

Keywords: discrete fractional mixed type sum-difference equation; boundary value problem; Banach space; existence and uniqueness; Banach contraction mapping principle

\section{Introduction}

For $a, b \in \mathbb{R}$, such that $b-a$ is a nonnegative integer, we define $\mathbb{N}_{a}=\{a, a+1, a+2, \ldots\}$ and $\mathbb{N}_{a}^{b}=\{a, a+1, \ldots, b\}$ throughout this paper. It is also worth noting that, in what follows, for any Banach-valued function $u$ defined on $\mathbb{N}_{a}$, we appeal to the convention $\sum_{s=k_{1}}^{k_{2}} u(s)=\theta$, where $k_{1}, k_{2} \in \mathbb{N}_{a}$ with $k_{1}>k_{2}$ and $\theta$ is the zero element of a given Banach space.

In this paper, we will consider the existence of a unique solution to the following discrete fractional mixed type sum-difference equation boundary value problem in the Banach space $E$ :

$$
\left\{\begin{array}{l}
\Delta^{\alpha} u(t)+f(t+\alpha-1, u(t+\alpha-1),(T u)(t),(S u)(t))=\theta, \quad t \in \mathbb{N}_{0}, \\
u(\alpha-n)=\Delta u(\alpha-n)=\Delta^{2} u(\alpha-n)=\cdots=\Delta^{n-2} u(\alpha-n)=\theta, \\
\Delta^{\alpha-1} u(\infty)=u_{\infty}
\end{array}\right.
$$

where $n-1<\alpha \leq n, n \in \mathbb{N}_{2}, \Delta^{\alpha}$ denotes the discrete Riemann-Liouville fractional difference of order $\alpha, f: \mathbb{N}_{\alpha-1} \times E \times E \times E \rightarrow E$ is continuous, $\theta$ represents the zero element of $E, \Delta^{\alpha-1} u(\infty)=\lim _{t \rightarrow+\infty} \Delta^{\alpha-1} u(t)=u_{\infty} \in E$ and

$$
(T u)(t)=\sum_{s=0}^{t} k(t, s) u(s+\alpha-1), \quad(S u)(t)=\sum_{s=0}^{\infty} h(t, s) u(s+\alpha-1),
$$

where $k: D \rightarrow \mathbb{R}, D=\left\{(t, s) \in \mathbb{N}_{0} \times \mathbb{N}_{0}: s \leq t\right\}, h: \mathbb{N}_{0} \times \mathbb{N}_{0} \rightarrow \mathbb{R}$.

O2014 Lv and Feng; licensee Springer. This is an Open Access article distributed under the terms of the Creative Commons Attribution License (http://creativecommons.org/licenses/by/2.0), which permits unrestricted use, distribution, and reproduction in any medium, provided the original work is properly cited. 
Discrete fractional calculus is a generalization of ordinary difference and summation on arbitrary order that can be non-integer, and it has gained considerable popularity due mainly to its demonstrated applications in describing some real-world phenomena $[1,2]$. Among all the topics, the branch of discrete fractional boundary value problems is currently undergoing active investigation; see, for example, [3-17] and the references therein.

Boundary value problems for differential equations in Banach spaces have been studied by many authors [18-36]. Especially for the study of nonlinear mixed type integrodifferential equations which arise from many nonlinear problems in science [36], a series of excellent results have been obtained in recent years [21, 23, 30-36].

On the other hand, it is well known that discrete analogues of differential equations can be very useful in applications $[37,38]$, in particular for using computer to simulate the behavior of solutions for certain dynamic equations. However, compared to continuous case, significantly less is known about discrete difference calculus in Banach spaces [3945]. Furthermore, as far as we know, the theory of discrete fractional mixed type sumdifference equations boundary value problems in Banach spaces is still a new research area. So, in this paper, we focus on this gap and provide some sufficient conditions for the existence and uniqueness of solutions to problem (1.1).

The remainder of this paper is organized as follows. Section 2 preliminarily presents some necessary basic knowledge for the theory of discrete fractional calculus in Banach spaces. In Section 3, the existence and uniqueness result for the solution to problem (1.1) will be established with the help of the contraction mapping principle. Finally, in Section 4, two concrete examples are provided to illustrate the possible applications of the established analytical result.

\section{Preliminaries}

In this section, we firstly present the definitions for the discrete Riemann-Liouville fractional difference and the discrete fractional sum for Banach-valued functions similar to the corresponding definitions for real-valued functions [46-49].

Definition 2.1 ([46]) For any $t$ and $v$, the falling factorial function is defined as

$$
t^{\underline{v}}=\frac{\Gamma(t+1)}{\Gamma(t+1-v)}
$$

provided that the right-hand side is well defined. We appeal to the convention that if $t+$ $1-v$ is a pole of the gamma function and $t+1$ is not a pole, then $t^{\underline{v}}=0$.

Definition 2.2 The $v$ th discrete fractional sum of a function $f: \mathbb{N}_{a} \rightarrow E$, for $v>0$, is defined by

$$
\Delta_{a}^{-v} f(t)=\frac{1}{\Gamma(v)} \sum_{s=a}^{t-v}(t-s-1)^{\frac{\nu-1}{f}} f(s), \quad t \in \mathbb{N}_{a+\nu} .
$$

Also, we define the trivial sum $\Delta_{a}^{-0} f(t)=f(t), t \in \mathbb{N}_{a}$. 
Definition 2.3 The $v$ th discrete Riemann-Liouville fractional difference of a function $f$ : $\mathbb{N}_{a} \rightarrow E$, for $v>0$, is defined by

$$
\Delta_{a}^{v} f(t)=\Delta^{n} \Delta_{a}^{-(n-v)} f(t), \quad t \in \mathbb{N}_{a+n-v},
$$

where $n$ is the smallest integer greater than or equal to $v$ and $\Delta^{n}$ is the $n$th order forward difference operator. If $v=n \in \mathbb{N}_{1}$, then $\Delta_{a}^{n} f(t)=\Delta^{n} f(t)$.

Remark 2.1 From Definitions 2.2 and 2.3, it is easy to see that $\Delta_{a}^{-v}$ maps functions defined on $\mathbb{N}_{a}$ to functions defined on $\mathbb{N}_{a+v}$ and $\Delta_{a}^{v}$ maps functions defined on $\mathbb{N}_{a}$ to functions defined on $\mathbb{N}_{a+n-v}$, where $n$ is the smallest integer greater than or equal to $v$. Also, it is worth reminding the reader that the $t$ in $\Delta_{a}^{v} f(t)$ (or $\Delta_{a}^{-v} f(t)$ ) represents an input for the function $\Delta_{a}^{v} f$ (or $\Delta_{a}^{-v} f$ ) and not for the function $f$. For ease of notation, throughout this paper we omit the subscript $a$ in $\Delta_{a}^{v} f(t)$ and $\Delta_{a}^{-v} f(t)$ when it does not lead to domain confusion and general ambiguity.

Now, we present the following two results, which are analogues to the ordinary case for the real-valued function.

Lemma 2.1 Let $f: \mathbb{N}_{a} \rightarrow E$ and $v, \mu>0$. Then

$$
\Delta_{a+\mu}^{-v} \Delta_{a}^{-\mu} f(t)=\Delta_{a}^{-v-\mu} f(t)=\Delta_{a+v}^{-\mu} \Delta_{a}^{-v} f(t), \quad t \in \mathbb{N}_{a+\mu+v} .
$$

Lemma 2.2 Let $f: \mathbb{N}_{a} \rightarrow E, v>0$ and $p$ be a positive integer. Then

$$
\Delta_{a}^{-v} \Delta^{p} f(t)=\Delta^{p} \Delta_{a}^{-v} f(t)-\sum_{i=1}^{p} \frac{(t-a)^{\underline{v-i}}}{\Gamma(v-i+1)} \Delta^{p-i} f(a) .
$$

Remark 2.2 Lemma 2.1 and Lemma 2.2 are natural analogues of Theorem 2.2 in [46] and Theorem 2.2 in [47] for real-valued functions. Their proofs are similar to the ordinary case. So, here we omit them. Additionally, by using Lemma 2.1, we can easily obtain the equality $\Delta^{v} \Delta^{-v} f(t)=f(t), v>0$ holds, for any Banach-valued function $f$.

At last, we need to state the following lemmas, which will be important in the sequel.

Lemma 2.3 Let $v>0$ and $f: \mathbb{N}_{a} \rightarrow E$. Then

$$
\begin{aligned}
\Delta_{a+n-v}^{-v} \Delta_{a}^{v} f(t)= & f(t)+c_{1}(t-a-n+v)^{\frac{v-1}{}} \\
& +c_{2}(t-a-n+v) \frac{v-2}{+}+\cdots+c_{n}(t-a-n+v) \frac{v-n}{n},
\end{aligned}
$$

where $c_{i} \in E, i=1,2, \ldots, n$, and $n$ is the smallest integer greater than or equal to $v$.

Proof By Definition 2.3, Lemma 2.1, Lemma 2.2 and Remark 2.2, we have

$$
\begin{aligned}
& \Delta_{a+n-v}^{-v} \Delta_{a}^{v} f(t) \\
& \quad=\left(\Delta_{a+n-v}^{-v} \Delta^{n} \Delta_{a}^{-(n-v)} f\right)(t)
\end{aligned}
$$




$$
\begin{aligned}
& =\left(\Delta^{n} \Delta_{a+n-v}^{-v} \Delta_{a}^{-(n-v)} f\right)(t)-\sum_{i=1}^{n} \frac{(t-a-n+v)^{\frac{v-i}{}}}{\Gamma(v-i+1)}\left[\left(\Delta^{n-i} \Delta_{a}^{-(n-v)} f\right)(a+n-v)\right] \\
& =f(t)-\sum_{i=1}^{n} \frac{(t-a-n+v) \frac{v-i}{\Gamma(v-i+1)}}{\left.\Gamma\left(\Delta^{n-i} \Delta_{a}^{-(n-v)} f\right)(a+n-v)\right] .}
\end{aligned}
$$

Setting $c_{i}=-\frac{\left(\Delta^{n-i} \Delta_{a}^{-(n-v)} f\right)(a+n-v)}{\Gamma(v-i+1)}, i=1,2, \ldots, n$; then we get (2.1). So the proof is complete.

Lemma 2.4 ([48]) Let $a \in \mathbb{R}$ and $\mu>0$ be given. Then

$$
\Delta(t-a) \underline{\mu}=\mu(t-a) \underline{\mu-1}
$$

for any $t$ for which both sides are well defined. Furthermore, for $n-1<v \leq n, n \in \mathbb{N}_{1}$ and $\mu \in \mathbb{R} \backslash\left(-\mathbb{N}_{1}\right)$,

$$
\Delta_{a+\mu}^{-v}(t-a) \underline{\mu}=\mu \underline{-v}(t-a) \stackrel{\mu+v}{\underline{n}}, \quad t \in \mathbb{N}_{a+\mu+v},
$$

and

$$
\Delta_{a+\mu}^{v}(t-a)^{\underline{\mu}}=\mu^{\underline{v}}(t-a) \stackrel{\mu-v}{\underline{ }}, \quad t \in \mathbb{N}_{a+\mu+n-v} .
$$

\section{Main results}

In this section, we establish the existence of a unique solution to problem (1.1). To accomplish this, we firstly list here the following conditions.

$\left(C_{1}\right)$ There exist constants $k^{*}$ and $h^{*}$ such that

$$
\begin{aligned}
& k^{*}=\sup _{t \in \mathbb{N}_{0}} \sum_{s=0}^{t}|k(t, s)|<+\infty \\
& h^{*}=\sup _{t \in \mathbb{N}_{0}} \frac{1}{1+(t+\alpha-1) \frac{\alpha-1}{2}} \sum_{s=0}^{\infty}|h(t, s)|\left[1+(s+\alpha-1)^{\left.\frac{\alpha-1}{}\right]<+\infty .}\right.
\end{aligned}
$$

$\left(\mathrm{C}_{2}\right) f^{*}=\sum_{t=\alpha-1}^{\infty}\|f(t, \theta, \theta, \theta)\|<+\infty$, and there exist nonnegative numbers $a, b, c$ and a function $p: \mathbb{N}_{\alpha-1} \rightarrow[0, \infty)$ with $p^{*}=\sum_{t=\alpha-1}^{\infty} p(t)\left(1+t^{\alpha-1}\right)<+\infty$ such that

$$
\|f(t, u, v, w)-f(t, \bar{u}, \bar{v}, \bar{w})\| \leq p(t)(a\|u-\bar{u}\|+b\|v-\bar{v}\|+c\|w-\bar{w}\|)
$$

for $t \in \mathbb{N}_{\alpha-1}, u, v, w, \bar{u}, \bar{v}, \bar{w} \in E$.

Next, we define

$$
X=\left\{u: \mathbb{N}_{\alpha-n} \rightarrow E \mid \sup _{t \in \mathbb{N}_{\alpha-n}} \frac{\|u(t)\|}{1+t \underline{\underline{\alpha-1}}}<+\infty\right\}
$$

equipped with the norm

$$
\|u\|_{X}=\sup _{t \in \mathbb{N}_{\alpha-n}} \frac{\|u(t)\|}{1+t^{\underline{\alpha-1}}} .
$$


Furthermore, by means of the linear functional analysis theory, we can easily prove that $\left(X,\|\cdot\|_{X}\right)$ is a Banach space.

Next, we state and prove the following lemmas, which will be used to establish the existence result of solutions to problem (1.1).

Lemma 3.1 If $\left(\mathrm{C}_{1}\right)$ and $\left(\mathrm{C}_{2}\right)$ hold, then, for any $u \in X$,

$$
\sum_{t=0}^{\infty}\|f(t+\alpha-1, u(t+\alpha-1),(T u)(t),(S u)(t))\| \leq p^{*}\left(a+b k^{*}+c h^{*}\right)\|u\|_{X}+f^{*} .
$$

Proof Setting $\bar{u}=\bar{v}=\bar{w}=\theta$ in $\left(C_{2}\right)$, we have

$$
\begin{gathered}
\|f(t, u, v, w)\| \leq p(t)(a\|u\|+b\|v\|+c\|w\|)+\|f(t, \theta, \theta, \theta)\|, \\
(t, u, v, w) \in \mathbb{N}_{\alpha-1} \times E \times E \times E .
\end{gathered}
$$

So, for any $u \in X, t \in \mathbb{N}_{0}$, using $\left(C_{2}\right)$ again produces

$$
\begin{aligned}
& \|f(t+\alpha-1, u(t+\alpha-1),(T u)(t),(S u)(t))\| \\
& \leq p(t+\alpha-1)(a\|u(t+\alpha-1)\|+b\|(T u)(t)\|+c\|(S u)(t)\|)+\|f(t+\alpha-1, \theta, \theta, \theta)\| \\
& =p(t+\alpha-1)\left[1+(t+\alpha-1)^{\frac{\alpha-1}{n}}\right]\left(a \frac{\|u(t+\alpha-1)\|}{1+(t+\alpha-1) \underline{\underline{\alpha-1}}}+b \frac{\|(T u)(t)\|}{1+(t+\alpha-1)^{\underline{\alpha-1}}}\right. \\
& \left.+c \frac{\|(S u)(t)\|}{1+(t+\alpha-1)^{\underline{\alpha-1}}}\right)+\|f(t+\alpha-1, \theta, \theta, \theta)\| \\
& \leq p(t+\alpha-1)\left[1+(t+\alpha-1)^{\left.\frac{\alpha-1}{2}\right]}\left(a\|u\|_{X}+b k^{*}\|u\|_{X}+c h^{*}\|u\|_{X}\right)\right. \\
& +\|f(t+\alpha-1, \theta, \theta, \theta)\| \text {. }
\end{aligned}
$$

Summating both sides of (3.2), we can get (3.1). The proof is completed.

Lemma 3.2 Let $h: \mathbb{N}_{0} \rightarrow E$ be given and $n-1<\alpha \leq n, n \in \mathbb{N}_{2}$. The unique solution of

$$
\left\{\begin{array}{l}
\Delta^{\alpha} u(t)+h(t)=\theta, \quad t \in \mathbb{N}_{0}, \\
u(\alpha-n)=\Delta u(\alpha-n)=\Delta^{2} u(\alpha-n)=\cdots=\Delta^{n-2} u(\alpha-n)=\theta, \\
\Delta^{\alpha-1} u(\infty)=u_{\infty},
\end{array}\right.
$$

is

$$
u(t)=\sum_{s=0}^{\infty} G(t, s) h(s)+\frac{u_{\infty}}{\Gamma(\alpha)} t^{\alpha-1}, \quad t \in \mathbb{N}_{\alpha-n},
$$

where

$$
G(t, s)=\frac{1}{\Gamma(\alpha)} \begin{cases}t^{\frac{\alpha-1}{2}}-(t-s-1)^{\frac{\alpha-1}{},} & s \in \mathbb{N}_{0}^{t-\alpha} \\ t^{\underline{\alpha-1}}, & s \in \mathbb{N}_{t-\alpha+1} .\end{cases}
$$


Proof Suppose that $u: \mathbb{N}_{\alpha-n} \rightarrow E$ satisfies the equation of problem (3.3), then Lemma 2.3 implies that

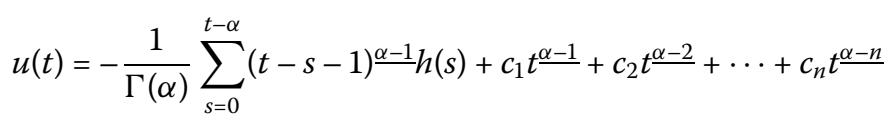

for some $c_{i} \in E, i=1,2, \ldots, n, t \in \mathbb{N}_{\alpha-n}$. By $u(\alpha-n)=\theta$, we get $c_{n}=\theta$.

Furthermore, in view of Lemma 2.4, we have

$$
\begin{aligned}
\Delta u(t)= & -\frac{1}{\Gamma(\alpha-1)} \sum_{s=0}^{t-(\alpha-1)}(t-s-1)^{\frac{\alpha-2}{h}} h(s) \\
& +c_{1}(\alpha-1) t^{\frac{\alpha-2}{2}}+c_{2}(\alpha-2) t^{\frac{\alpha-3}{2}}+\cdots+c_{n-1}(\alpha-n+1) t^{\frac{\alpha-n}{}} .
\end{aligned}
$$

Substituting $\Delta u(\alpha-n)=\theta$ in (3.5) gives $c_{n-1}=\theta$.

Repeating the above steps with $\Delta^{2} u(\alpha-n)=\cdots=\Delta^{n-2} u(\alpha-n)=\theta$, we can get

$$
c_{n-2}=c_{n-3}=\cdots=c_{2}=\theta .
$$

Therefore,

$$
u(t)=-\frac{1}{\Gamma(\alpha)} \sum_{s=0}^{t-\alpha}(t-s-1)^{\frac{\alpha-1}{h}} h(s)+c_{1} t^{\frac{\alpha-1}{1}}, \quad t \in \mathbb{N}_{\alpha-n} .
$$

By virtue of Lemma 2.4 again, we have

$$
\Delta^{\alpha-1} u(t)=-\sum_{s=0}^{t-1} h(s)+c_{1} \Gamma(\alpha), \quad t \in \mathbb{N}_{0}
$$

Using the condition $\Delta^{\alpha-1} u(\infty)=u_{\infty}$ in (3.7), we obtain

$$
c_{1}=\frac{1}{\Gamma(\alpha)}\left(\sum_{s=0}^{\infty} h(s)+u_{\infty}\right)
$$

Now, substitution of $c_{1}$ into (3.6) gives

$$
\begin{aligned}
u(t) & =-\frac{1}{\Gamma(\alpha)} \sum_{s=0}^{t-\alpha}(t-s-1)^{\frac{\alpha-1}{h}} h(s)+\frac{1}{\Gamma(\alpha)} \sum_{s=0}^{\infty} t^{\frac{\alpha-1}{h}} h(s)+\frac{u_{\infty}}{\Gamma(\alpha)} t^{\underline{\alpha-1}} \\
& =\sum_{s=0}^{\infty} G(t, s) h(s)+\frac{u_{\infty}}{\Gamma(\alpha)} t^{\frac{\alpha-1}{2}}, \quad t \in \mathbb{N}_{\alpha-n},
\end{aligned}
$$

where $G(t, s)$ is defined by (3.4). The proof is complete.

Remark 3.1 From the expression of $G(t, s)$, we can easily find that $G(t, s) \geq 0$ and $\frac{G(t, s)}{1+t^{\frac{\alpha-1}{}}}<$ $\frac{1}{\Gamma(\alpha)}$ for $(t, s) \in \mathbb{N}_{n-\alpha} \times \mathbb{N}_{0}$.

With the above auxiliary results in hand, we now establish the main result as follows. 
Theorem 3.1 If $\left(\mathrm{C}_{1}\right),\left(\mathrm{C}_{2}\right)$ hold and

$$
\sigma=\frac{p^{*}\left(a+b k^{*}+c h^{*}\right)}{\Gamma(\alpha)}<1
$$

then problem (1.1) has a unique solution $u$ in $X$.

Proof Define an operator $\mathcal{F}: X \rightarrow X$ by

$$
(\mathcal{F} u)(t)=\sum_{s=0}^{\infty} G(t, s) f(s+\alpha-1, u(s+\alpha-1),(T u)(s),(S u)(s))+\frac{u_{\infty}}{\Gamma(\alpha)} t^{\underline{\alpha-1}}
$$

where $t \in \mathbb{N}_{\alpha-n}$, and due to Lemma 3.1, we have

$$
\begin{aligned}
\frac{\|(\mathcal{F} u)(t)\|}{1+t^{\alpha-1}} & \leq \sum_{s=0}^{\infty} \frac{G(t, s)}{1+t^{\underline{\alpha-1}}}\|f(s+\alpha-1, u(s+\alpha-1),(T u)(s),(S u)(s))\|+\frac{\left\|u_{\infty}\right\| t^{\frac{\alpha-1}{}}}{\Gamma(\alpha)\left(1+t^{\frac{\alpha-1}{}}\right)} \\
& \leq \frac{1}{\Gamma(\alpha)}\left\{p^{*}\left(a+b k^{*}+c h^{*}\right)\|u\|_{X}+f^{*}+\left\|u_{\infty}\right\|\right\} \\
& =\sigma\|u\|_{X}+\varrho, \quad t \in \mathbb{N}_{\alpha-n} .
\end{aligned}
$$

Therefore,

$$
\|\mathcal{F} u\|_{X} \leq \sigma\|u\|_{X}+\varrho, \quad u \in X
$$

here $\varrho=\left(f^{*}+\left\|u_{\infty}\right\|\right) / \Gamma(\alpha)$ and $\sigma$ is defined by (3.8). So, the operator $\mathcal{F}$ is well defined. Furthermore, from Lemma 3.2, we can transform problem (1.1) as an operator equation $u=\mathcal{F} u$, and it is clear to see that $u$ is a solution of problem (1.1) is equivalent to a fixed point of $\mathcal{F}$.

Next, for any $u, v \in X$, we denote

$$
\begin{aligned}
A(u, v)(t)= & f(t+\alpha-1, u(t+\alpha-1),(T u)(t),(S u)(t)) \\
& -f(t+\alpha-1, v(t+\alpha-1),(T v)(t),(S v)(t)), \quad t \in \mathbb{N}_{0} .
\end{aligned}
$$

In view of $\left(C_{2}\right)$, we have

$$
\begin{aligned}
& \frac{\|(\mathcal{F} u)(t)-(\mathcal{F} v)(t)\|}{1+t^{\underline{\alpha-1}}} \\
& \leq \sum_{s=0}^{\infty} \frac{G(t, s)}{1+t^{\alpha-1}}\|A(u, v)(s)\| \\
& \leq \frac{1}{\Gamma(\alpha)} \sum_{s=0}^{\infty}[p(s+\alpha-1)(a\|u(s+\alpha-1)-v(s+\alpha-1)\|+b\|(T u)(s)-(T v)(s)\| \\
& \quad+c\|(S u)(s)-(S v)(s)\|)] \\
& \leq \frac{1}{\Gamma(\alpha)} \sum_{s=0}^{\infty}\left[p(s+\alpha-1)\left[1+(s+\alpha-1)^{\frac{\alpha-1}{}}\right]\right.
\end{aligned}
$$




$$
\begin{aligned}
& \left.\times\left(a\|u-v\|_{X}+b k^{*}\|u-v\|_{X}+c h^{*}\|u-v\|_{X}\right)\right] \\
\leq & \frac{1}{\Gamma(\alpha)} p^{*}\left(a+b k^{*}+c h^{*}\right)\|u-v\|_{X} \\
= & \sigma\|u-v\|_{X} .
\end{aligned}
$$

So we get

$$
\|\mathcal{F} u-\mathcal{F} v\|_{X} \leq \sigma\|u-v\|_{X},
$$

which, together with the assumption that $\sigma<1$, implies that $\mathcal{F}$ is a contraction mapping. By means of the Banach contraction mapping principle, we get that $\mathcal{F}$ has a unique fixed point in $E$; that is problem (1.1) has a unique solution. This completes the proof.

\section{Examples}

In this section, we illustrate the possible application of the above established analytical result with the following two concrete examples.

Example 4.1 Consider the following problem:

$$
\left\{\begin{array}{l}
\Delta^{7 / 2} u_{n}(t)+\frac{3^{-(t+1)}}{n\left[1+(t+5 / 2)^{5 / 2}\right]^{2}} \sin \left[n^{2}(t+5 / 2)+u_{n}(t+5 / 2)\right] \\
\quad+\frac{2^{-(t+1)}}{(n+2)^{2}\left[1+(t+5 / 2)^{5 / 2}\right]^{3}} \ln \left\{1+\left[\sum_{s=0}^{t} \frac{1}{(t+s+2)^{2}} u_{3 n}(s+5 / 2)\right]^{2}\right\} \\
\quad+\frac{e^{(-(t+1)}}{\sqrt{n}\left[3+\sin (t+5 / 2)+(t+5 / 2) \frac{5 / 2}{2}\right]} \\
\quad \times\left\{\sum_{s=0}^{\infty} \frac{\cos \left(t^{2} s\right)}{(s+2)^{2}\left[1+(s+5 / 2)^{5 / 2}\right]} u_{n+1}(s+5 / 2)\right\}=0, \quad t \in \mathbb{N}_{0}, \\
u_{n}(-1 / 2)=\Delta u_{n}(-1 / 2)=\Delta^{2} u_{n}(-1 / 2)=0, \\
\Delta^{5 / 2} u_{n}(\infty)=\frac{1}{n !}, \quad n=1,2,3, \ldots
\end{array}\right.
$$

Conclusion Problem (4.1) has a unique solution $\left\{u_{n}(t)\right\}$ such that $u_{n}(t) \rightarrow 0$ as $n \rightarrow \infty$ for $t \in \mathbb{N}_{-1 / 2}$.

Proof Let $E=c_{0}=\left\{u=\left(u_{1}, u_{2}, \ldots, u_{n}, \ldots\right): u_{n} \rightarrow 0\right\}$. Evidently, $(E,\|\cdot\|)$ is a Banach space with the norm $\|u\|=\sup _{n}\left|u_{n}\right|$ for any $u \in E$. Then the infinite discrete fractional difference system (4.1) can be regarded as a boundary value problem of the form (1.1) in the Banach space $E$. In this situation, $\alpha=7 / 2, \theta=(0,0, \ldots, 0, \ldots) \in E, u_{\infty}=(1,1 / 2 !, \ldots, 1 / n !, \ldots) \in E$,

$$
k(t, s)=\frac{1}{(t+s+2)^{2}}, \quad h(t, s)=\frac{\cos \left(t^{2} s\right)}{(s+2)^{2}\left[1+(s+5 / 2)^{\frac{5 / 2}{2}}\right]},
$$

and $f=\left(f_{1}, f_{2}, \ldots, f_{n}, \ldots\right)$, in which

$$
\begin{aligned}
f_{n}(t, u, v, w)= & \frac{3^{-(t-3 / 2)}}{n\left[1+t \frac{5 / 2}{2}\right]^{2}} \sin \left(n^{2} t+u_{n}\right)+\frac{2^{-(t-3 / 2)}}{(n+2)^{2}\left[1+t \frac{5 / 2}{3}\right]^{3}} \ln \left(1+v_{3 n}^{2}\right) \\
& +\frac{e^{-(t-3 / 2)}}{\sqrt{n}\left[3+\sin t+t \frac{5 / 2}{}\right]} w_{n+1},
\end{aligned}
$$

where $t \in \mathbb{N}_{5 / 2}$ and $u=\left(u_{1}, u_{2}, \ldots, u_{n}, \ldots\right), v=\left(v_{1}, v_{2}, \ldots, v_{n}, \ldots\right), w=\left(w_{1}, w_{2}, \ldots, w_{n}, \ldots\right) \in E$. From the expression of $f_{n}$, it is easy to see that $f: \mathbb{N}_{5 / 2} \times E \times E \times E \rightarrow E$ is continuous. 
Furthermore, for any $t \in \mathbb{N}_{5 / 2}, u, v, w, \bar{u}, \bar{v}, \bar{w} \in E$, we have

$$
\begin{aligned}
&\left|f_{n}(t, u, v, w)-f_{n}(t, \bar{u}, \bar{v}, \bar{w})\right| \\
& \leq \frac{3^{-(t-3 / 2)}}{n\left[1+t \frac{5 / 2}{2}\right]^{2}}\left|\sin \left(n^{2} t+u_{n}\right)-\sin \left(n^{2} t+\bar{u}_{n}\right)\right| \\
&+\frac{2^{-(t-3 / 2)}}{(n+2)^{2}[1+t \underline{5 / 2}]^{3}}\left|\ln \left(1+v_{3 n}^{2}\right)-\ln \left(1+\bar{v}_{3 n}^{2}\right)\right| \\
&+\frac{e^{-(t-3 / 2)}}{\sqrt{n}\left[3+\sin t+t \frac{5 / 2}{3}\right]}\left|w_{n+1}-\bar{w}_{n+1}\right| \\
& \leq \frac{3^{-(t-3 / 2)}}{n\left[1+t \frac{5 / 2}{2}\right]^{2}}\left|u_{n}-\bar{u}_{n}\right|+\frac{2^{-(t-3 / 2)}}{(n+2)\left[1+t^{5 / 2}\right]^{3}}\left|v_{3 n}-\bar{v}_{3 n}\right| \\
&+\frac{e^{-(t-3 / 2)}}{\sqrt{n}[3+\sin t+t \underline{5 / 2}]}\left|w_{n+1}-\bar{w}_{n+1}\right| \\
& \leq \frac{2^{-(t-3 / 2)}}{1+t \frac{5 / 2}{1}}\left[\left|u_{n}-\bar{u}_{n}\right|+1 / 3\left|v_{3 n}-\bar{v}_{3 n}\right|+\left|w_{n+1}-\bar{w}_{n+1}\right|\right]
\end{aligned}
$$

and therefore,

$$
\|f(t, u, v, w)-f(t, \bar{u}, \bar{v}, \bar{w})\| \leq \frac{2^{-(t-3 / 2)}}{1+t \underline{5 / 2}}[\|u-\bar{u}\|+1 / 3\|v-\bar{v}\|+\|w-\bar{w}\|],
$$

where $a=c=1, b=\frac{1}{3}, p(t)=\frac{2^{-(t-3 / 2)}}{1+t^{\frac{5 / 2}{2}}}$, which imply that $\left(\mathrm{C}_{2}\right)$ holds together with the following facts:

$$
p^{*}=\sum_{t=5 / 2}^{\infty} p(t)\left(1+t^{5 / 2}\right)=\sum_{t=5 / 2}^{\infty} 2^{-(t-3 / 2)}=1<\infty
$$

and

$$
f^{*}=\sum_{s=5 / 2}^{\infty}\|f(t, \theta, \theta, \theta)\| \leq \sum_{s=5 / 2}^{\infty} 3^{-(t-3 / 2)}=1 / 2<\infty
$$

On the other hand, we can verify that

$$
\begin{aligned}
k^{*} & =\sup _{t \in \mathbb{N}_{0}} \sum_{s=0}^{t} \frac{1}{(t+s+2)^{\underline{2}}}=\sup _{t \in \mathbb{N}_{0}} \frac{1}{2(t+1)}=\frac{1}{2}<\infty, \\
h^{*} & =\sup _{t \in \mathbb{N}_{0}} \frac{1}{1+(t+5 / 2)^{\frac{5 / 2}{2}}} \sum_{s=0}^{\infty} \frac{\left|\cos \left(t^{2} s\right)\right|\left[1+(s+5 / 2)^{\frac{5 / 2}{2}}\right]}{\left[1+(s+5 / 2)^{\frac{5 / 2}{2}}\right](s+2)^{\underline{2}}} \\
& \leq \sup _{t \in \mathbb{N}_{0}} \frac{1}{1+(t+5 / 2)^{\frac{5 / 2}{}}} \sum_{s=0}^{\infty} \frac{1}{(s+2)^{\underline{2}}} \\
& \leq \frac{1}{1+\Gamma(7 / 2)}<\frac{1}{4}<\infty .
\end{aligned}
$$

So $\left(C_{1}\right)$ is also satisfied. Finally, by a simple calculation, we can obtain

$$
\sigma=\frac{p^{*}\left(a+b k^{*}+c h^{*}\right)}{\Gamma(\alpha)} \leq \frac{(1+1 / 6+1 / 4)}{\Gamma(7 / 2)}<0.43<1 .
$$


Thus, all the conditions of Theorem 3.1 are satisfied and our conclusion follows from Theorem 3.1.

Example 4.2 Consider the following problem:

$$
\left\{\begin{array}{l}
\Delta^{7 / 3} \omega(t, x)+\frac{2^{-(t+1)}}{4\left[1+(t+4 / 3)^{4 / 3}\right]} \cos [\omega(t+4 / 3, x)] \\
\quad+\frac{3^{-(t+1)}}{e^{2}\left[1+\left(t+4 / 3 \underline{4 / 3}^{-/ 3}\right.\right.}\left[\sum_{s=0}^{t} \frac{1}{(t+s+2)^{2}} \omega(s+4 / 3, x)\right] \\
\quad+\frac{e^{-(t+1)}}{e^{3}\left[2+\cos (t+4 / 3)+(t+4 / 3)^{\underline{4 / 3}}\right]}\left\{\sum_{s=0}^{\infty} \frac{\sin \left(t+e^{s}\right)}{(s+2)^{2}\left[1+(s+4 / 3)^{4 / 3}\right]} \omega(s+4 / 3, x)\right\}=0 \\
t \in \mathbb{N}_{0}, x \in[0,1] \\
\omega(-2 / 3, x)=\Delta_{t} \omega(-2 / 3, x)=0, \quad \Delta^{4 / 3} \omega(\infty, x)=x^{2}
\end{array}\right.
$$

Here, $\Delta^{7 / 3} \omega(t, x)$ represents the discrete Riemann-Liouville fractional difference of order $7 / 3$ for the function $\omega(t, x)$ with respect to its first variable $t$.

Conclusion Problem (4.2) has a unique solution $\omega: \mathbb{N}_{-2 / 3} \times[0,1] \rightarrow \mathbb{R}$ such that for each given $t \in \mathbb{N}_{-2 / 3}, \omega(t, x)$ is continuous for $x \in[0,1]$.

Proof Let $E=C[0,1]=\{g:[0,1] \rightarrow \mathbb{R}$ is continuous $\}$; then $(E,\|\cdot\|)$ is a Banach space equipped with the norm $\|g\|=\sup _{x \in[0,1]}|g(x)|, g \in E$. Define $u: \mathbb{N}_{-2 / 3} \rightarrow E$ by $u(t)=\omega(t, \cdot) \in$ $E$; then the discrete fractional partial difference system (4.2) can be transformed into the form of problem (1.1), where $\theta=0, u_{\infty}=x^{2}$,

$$
k(t, s)=\frac{1}{(t+s+2)^{2}}, \quad h(t, s)=\frac{\sin \left(t+e^{s}\right)}{(s+2)^{2}[1+(s+4 / 3) \underline{4 / 3}]},
$$

and

$$
f(t, u, v, w)=\frac{2^{-(t-1 / 3)}}{4[1+t \underline{4 / 3}]} \cos u+\frac{3^{-(t-1 / 3)}}{e^{2}[1+t \underline{4 / 3}]} v+\frac{e^{-(t-1 / 3)}}{e^{3}[2+\cos t+t \underline{4 / 3}]} w
$$

for $(t, u, v, w) \in \mathbb{N}_{4 / 3} \times E \times E \times E$. It is obvious that $f$ is continuous.

Choosing $a=\frac{1}{4}, b=\frac{1}{e^{2}}, c=\frac{1}{e^{3}}$ and $p(t)=\frac{2^{-(t-1 / 3)}}{1+t \frac{4 / 3}{3}}, t \in \mathbb{N}_{4 / 3}$; then we can verify that $p^{*}=1$, $f^{*}<1 / 4, k^{*}=1 / 2, h^{*}<0.4566, \sigma<0.3631$ and

$$
\|f(t, u, v, w)-f(t, \bar{u}, \bar{v}, \bar{w})\| \leq p(t)(a\|u-\bar{u}\|+b\|v-\bar{v}\|+c\|w-\bar{w}\|)
$$

holds for any $t \in \mathbb{N}_{4 / 3}, u, v, w, \bar{u}, \bar{v}, \bar{w} \in E$.

Clearly, all the conditions of Theorem 3.1 are fulfilled. Therefore, we can conclude that problem (4.2) has a unique solution.

Competing interests

The authors declare that they have no competing interests.

Authors' contributions

The authors declare that the study was realized in collaboration with the same responsibility. All authors read and approved the final manuscript. 


\section{Acknowledgements}

The authors sincerely thank the reviewers for their valuable suggestions and useful comments that have led to the present improved version of the original manuscript. The research is supported by the National Natural Science Foundation of China 11261032, the Longdong University Grant XYZK-1207 and XYZK-1402.

\section{Received: 23 April 2014 Accepted: 2 July 2014 Published: 22 Jul 2014}

\section{References}

1. Wu, G, Baleanu, D: Discrete fractional logistic map and its chaos. Nonlinear Dyn. 75, 283-287 (2014)

2. AtıcI, F, Şengül, S: Modeling with fractional difference equations. J. Math. Anal. Appl. 369, 1-9 (2010)

3. AtıI, F, Eloe, P: Two-point boundary value problems for finite fractional difference equations. J. Differ. Equ. Appl. 17, 445-456 (2011)

4. Goodrich, C: Solutions to a discrete right-focal fractional boundary value problem. Int. J. Differ. Equ. 5, 195-216 (2010)

5. Goodrich, C: Continuity of solutions to discrete fractional initial value problems. Comput. Math. Appl. 59, 3489-3499 (2010)

6. Goodrich, C: Existence and uniqueness of solutions to a fractional difference equation with nonlocal conditions. Comput. Math. Appl. 61, 191-202 (2011)

7. Goodrich, C: Existence of a positive solution to a system of discrete fractional boundary value problems. Appl. Math. Comput. 217, 4740-4753 (2011)

8. Goodrich, C: On a discrete fractional three-point boundary value problem. J. Differ. Equ. Appl. 18, 397-415 (2012)

9. Goodrich, C: On discrete sequential fractional boundary value problems. J. Math. Anal. Appl. 385, 111-124 (2012)

10. Goodrich, C: On semipositone discrete fractional boundary value problems with non-local boundary conditions. J. Differ. Equ. Appl. 19, 1758-1780 (2013)

11. Dahal, R, Duncan, D, Goodrich, C: Systems of semipositone discrete fractional boundary value problems. J. Differ. Equ. Appl. 20, 473-491 (2014)

12. Holm, M: Solutions to a discrete, nonlinear, $(N-1,1)$ fractional boundary value problem. Int. J. Dyn. Syst. Differ. Equ. 3 , 267-287 (2011)

13. Pan, Y, Han, Z, Sun, S, Hou, C: The existence of solutions to a class of boundary value problems with fractional difference equations. Adv. Differ. Equ. 2013, 275 (2013)

14. Ferreira, R: Existence and uniqueness of solution to some discrete fractional boundary value problems of order less than one. J. Differ. Equ. Appl. 19, 712-718 (2013)

15. Chen, F, Zhou, Y: Existence and Ulam stability of solutions for discrete fractional boundary value problem. Discrete Dyn. Nat. Soc. 2013, Article ID 459161 (2013)

16. Lv, W: Existence of solutions for discrete fractional boundary value problems with a $p$-Laplacian operator. Adv. Differ Equ. 2012, 163 (2012)

17. $L v, W$ : Solvability for discrete fractional boundary value problems with a $p$-Laplacian operator. Discrete Dyn. Nat. Soc. 2013, Article ID 679290 (2013)

18. Liu, Y: Boundary value problems for second order differential equations on unbounded domains in a Banach space. Appl. Math. Comput. 135, 569-583 (2003)

19. Zhang, $X$, Feng, $M, G e, W$ : Existence results for nonlinear boundary-value problems with integral boundary conditions in Banach spaces. Nonlinear Anal. 69, 3310-3321 (2008)

20. Zhang, X, Feng, $M, G e, W$ : Existence and nonexistence of positive solutions for a class of $n$ th-order three-point boundary value problems in Banach spaces. Nonlinear Anal. 70, 584-597 (2009)

21. Zhang, $X$, Feng, $M, G e, W$ : Existence of solutions of boundary value problems with integral boundary conditions for second-order impulsive integro-differential equations in Banach spaces. J. Comput. Appl. Math. 233, 1915-1926 (2010)

22. Feng, $M, J i, D, G e, W$ : Positive solutions for a class of boundary-value problem with integral boundary conditions in Banach spaces. J. Comput. Appl. Math. 222, 351-363 (2008)

23. Feng, M, Pang, H: A class of three-point boundary-value problems for second-order impulsive integro-differential equations in Banach spaces. Nonlinear Anal. 70, 64-82 (2009)

24. Chen, $\mathrm{H}$, Li, P: Existence of solutions of three-point boundary value problems in Banach spaces. Math. Comput. Model. 49, 780-788 (2009)

25. Chen, $\mathrm{H}, \mathrm{ZhaO}$, Y: Triple positive solutions for nonlinear boundary value problems in Banach space. Comput. Math. Appl. 58, 1780-1787 (2009)

26. Jiang, W, Wang, B: Positive solutions for second-order multi-point boundary value problems in Banach spaces. Electron. J. Differ. Equ. 2011, 22 (2011)

27. Hao, $X, L i u, L, W u, Y, X u, N$ : Multiple positive solutions for singular $n$ th-order nonlocal boundary value problems in Banach spaces. Comput. Math. Appl. 61, 1880-1890 (2011)

28. Su, X: Solutions to boundary value problem of fractional order on unbounded domains in a Banach space. Nonlinear Anal. 74, 2844-2852 (2011)

29. Guo, D, Lakshmikantham, V: Multiple solutions of two-point boundary value problems of ordinary differential equations in Banach spaces. J. Math. Anal. Appl. 129, 211-222 (1988)

30. Guo, D: A boundary value problem for $n$ th-order integro-differential equations in a Banach space. Appl. Math Comput. 136, 571-592 (2003)

31. Guo, D: Multiple positive solutions of a boundary value problem for $n$ th-order impulsive integro-differential equations in a Banach space. Nonlinear Anal. 56, 985-1006 (2004)

32. Guo, D: Multiple positive solutions of a boundary value problem for $n$ th-order impulsive integro-differentia equations in Banach spaces. Nonlinear Anal. 63, 618-641 (2005)

33. $\mathrm{Xu}, \mathrm{Y}, \mathrm{Zhang}, \mathrm{H}$ : Multiple positive solutions of a boundary value problem for a class of $2 n$ th-order $m$-point singular integro-differential equations in Banach spaces. Appl. Math. Comput. 214, 607-617 (2009)

34. $\mathrm{Xu}, \mathrm{Y}, \mathrm{Zhang}, \mathrm{H}$ : Multiple positive solutions of a $m$-point boundary value problem for 2 nth-order singular integro-differential equations in Banach spaces. Nonlinear Anal. 70, 3243-3253 (2009) 
35. Zhang, L, Ahmad, B, Wang, G, Agarwal, R: Nonlinear fractional integro-differential equations on unbounded domains in a Banach space. J. Comput. Appl. Math. 249, 51-56 (2013)

36. Lakshmikantham, V: Some problems in integro-differential equations of Volterra type. J. Integral Equ. 10, 137-146 (1985)

37. Hilscher, R, Zeidan, V: Nonnegativity and positivity of quadratic functionals in discrete calculus of variations: survey. J. Differ. Equ. Appl. 11, 857-875 (2005)

38. Kelley, W, Peterson, A: Difference Equations: An Introduction with Applications. Academic Press, New York (1991)

39. Agarwal, R, O'Regan, D: Difference equations in Banach spaces. J. Aust. Math. Soc. A 64, $277-284$ (1998)

40. Agarwal, R, O'Regan, D: A fixed-point approach for nonlinear discrete boundary value problems. Comput. Math. Appl. 36, 115-121 (1998)

41. González, C, Jiménez-Melado, A: An application of Krasnoselskii fixed point theorem to the asymptotic behavior of solutions of difference equations in Banach spaces. J. Math. Anal. Appl. 247, 290-299 (2000)

42. Tabor, J: Oscillation of linear difference equations in Banach spaces. J. Differ. Equ. 192, 170-187 (2003)

43. Bay, N, Phat, V: Stability analysis of nonlinear retarded difference equations in Banach spaces. Comput. Math. Appl. 45 951-960 (2003)

44. González, C, Jiménez-Melado, A: Set-contractive mappings and difference equations in Banach spaces. Comput. Math. Appl. 45, 1235-1243 (2003)

45. Agarwal, R, Thompson, H, Tisdell, C: Difference equations in Banach spaces. Comput. Math. Appl. 45, 1437-1444 (2003)

46. Atıcı, F, Eloe, P: A transform method in discrete fractional calculus. Int. J. Differ. Equ. 2, 165-176 (2007)

47. AtıCl, F, Eloe, P: Initial value problems in discrete fractional calculus. Proc. Am. Math. Soc. 137, 981-989 (2009)

48. Holm, M: Sum and difference compositions in discrete fractional calculus. CUBO 13, 153-184 (2011)

49. Miller, K, Ross, B: Fractional difference calculus. In: Proceedings of the International Symposium on Univalent Functions, Fractional Calculus and Their Applications, pp. 139-152. Nihon University, Koriyama (1989)

10.1186/1687-1847-2014-184

Cite this article as: Lv and Feng: Nonlinear discrete fractional mixed type sum-difference equation boundary value problems in Banach spaces. Advances in Difference Equations 2014, 2014:184

\section{Submit your manuscript to a SpringerOpen ${ }^{\ominus}$ journal and benefit from:}

- Convenient online submission

- Rigorous peer review

- Immediate publication on acceptance

Open access: articles freely available online

- High visibility within the field

- Retaining the copyright to your article 